\title{
Near-Field Optical Imaging of a Porous Au Film: Influences of Topographic Artifacts and Surface Plasmons
}

\author{
Yu-Hsuan Lin • Benjamin Händel • Hung Ji Huang • \\ Hsiang-An Chen • Yung-Fu Chen • Heh-Nan Lin • \\ Din Ping Tsai
}

Received: 4 May 2012 / Accepted: 31 May 2012 /Published online: 14 June 2012

(C) Springer Science+Business Media, LLC 2012

\begin{abstract}
In this work, near-field scanning optical microscopy is employed to study a porous Au film and the direct observation of topographic artifacts and surface plasmon influences is revealed. Under tip illumination, topographic artifacts are found to be present in a reflection mode optical image but not in a transmission mode image. A simple algorithm is used for filtering the topographic artifacts and extracting a correct near-field optical image approximately. On the other hand, surface plasmon influences are present in both modes. By using three exciting wavelengths of 488 , 647.1 , and $520.8 \mathrm{~nm}$, it is confirmed that a suitable wavelength should be chosen for avoiding the surface plasmon interference in a near-field optical investigation of morphological or material dielectric contrast. Finally, plasmonic or nonplasmonic regions on the porous Au film can be identified from the observed optical intensity variation in the
\end{abstract}

Y.-H. Lin • B. Händel • H. J. Huang • D. P. Tsai $(\bowtie)$

Instrument Technology Research Center,

National Applied Research Laboratories,

Hsinchu 30076, Taiwan

e-mail: dptsai@phys.ntu.edu.tw

Y.-H. Lin • Y.-F. Chen

Department of Electrophysics, National Chiao Tung University,

Hsinchu 30010, Taiwan

H.-A. Chen $\cdot$ H.-N. Lin

Department of Materials Science and Engineering,

National Tsing Hua University,

Hsinchu 30013, Taiwan

D. P. Tsai

Department of Physics, National Taiwan University,

Taipei 10617, Taiwan

D. P. Tsai

Research Center for Applied Sciences, Academia Sinica,

Taipei 115, Taiwan optical images obtained at incident polarizations of $0^{\circ}$, $90^{\circ}$, and $45^{\circ}$.

Keywords Near-field scanning optical microscopy · Porous gold film · Topographic artifact · Surface plasmon

\section{Introduction}

Near-field scanning optical microscopy (NSOM) provides us with eyes for observing the interesting nanoworld by means of its high-spatial resolution [1-4]. It is a powerful tool and has been widely used in nanophotonics, such as superresolution optical storage [5-7], surface-enhanced Raman scattering, metamaterials, and plasmonics [8-11]. In an aperture-type NSOM (a-NSOM), a coated fiber probe kept in the near-field region of the sample surface is used to depict the optical contrast of the sample without diffraction limit by converting the evanescent field into propagating field, and the topographic information of the sample can be obtained simultaneously by force feedback detection. The resolutions of a-NSOM and topographic images depend on the aperture and shape size of the probe apex, respectively. Different kinds of NSOM configuration and operating modes provide different optical information and physical interaction with the sample. Hence, an appropriate measurement mechanism of NSOM befitting the optical properties of the sample needs to be chosen [12].

Unfortunately, artifacts in NSOM measurements are not uncommon as has been explained by Hecht et al. [13]. The near-field optical image may be dominated by topographic features because of the "z-motion" operating mode (constant gap mode, CGM) and thus seriously mislead the estimations of resolution capabilities and near-field optical results [14-18]. One of the proposed solutions for this serious 
problem in NSOM is the use of constant height mode (CHM). However, only few commercial instruments offer this function and are prone to the risk that the probe collides with a large piece of dust on the sample during scanning. Besides, both the resolutions of topography and optical image in CHM are much lower than in CGM and there will be even the possibility that the probe can leave away the near-field region during the scan because of sample's tilt or roughness. The second proposed solution to avoid the artifacts is the use of "on-axis" measurement mode such as transmission mode with angle smaller than the critical one. This solution is useful in CGM yet limited by the necessity for the sample to be transparent. Yet a single kind of operating mode will not satisfy the various demands of different research purposes.

In addition to the topographic artifact caused by $z$-motion influence to the near-field images of the sample, the surface plasmon (SP) effect induced by a metallic structure also plays an important role $[19,20]$. SPs are oscillations of coherent free electrons at a metal-dielectric interface and these electromagnetic (EM) surface waves are usually called surface plasmon polaritons (SPPs) when propagating along the interface. They can be excited by light with well-known couplers such as gratings, attenuated total reflection devices, or metallic films with corrugated surface. SPs can also be localized or confined in a subwavelength region via patterned structures or a random media system such as nanoantenna, metallic holes, metallic particles or super-RENS [21-30], and are called localized surface plasmons (LSPs). This phenomenon has wide range of applications because of its superresolution and light enhancement characteristics. However, in pure near-field optical inspection, SPPs or LSPs may sometimes be an unwanted phenomenon because it masks the optical properties of the sample. The native optical details such as material dielectric contrasts and morphological contrasts in a near-field image are covered by new signals originated in the interplay of complex SP behaviors. This phenomenon cannot be treated as an artifact effect because the SPs still are of an optical origin even when "masking" part of the information of the sample.

In this work, an NSOM study of a porous Au film has been carried out to investigate topographic artifacts and surface plasmon influences on the optical images. Using the NSOM fiber tip as the illumination source, topographic artifacts are found to be present in an off-axis reflection mode (RM) image but not in an on-axis transmission mode (TM) image. A simple algorithm has been used for filtering the topographic artifacts and extracting the correct optical information approximately. On the other hand, surface plasmon influences are present in both modes. A series of TM measurements are conducted to clarify individual behavior of the plasmonic signals in the optical images by changing the incident light wavelength and polarization. This study supplies significant information for the nano-optical inspections and related applications.

\section{Sample Preparation and Experimental Setup}

In our experiment, the porous $\mathrm{Au}$ film was prepared by using a de-alloying method [31]. A quartz substrate was washed successively in acetone, ethanol and deionized water. A Ti adhesion layer $(\sim 1 \mathrm{~nm})$ was first deposited on the substrate by electron beam evaporation, and $20 \mathrm{~nm}$ thick $\mathrm{Au}$ and $20 \mathrm{~nm}$ thick $\mathrm{Cu}$ were subsequently deposited. The sample was then annealed at $200{ }^{\circ} \mathrm{C}$ for $6 \mathrm{~h}$ in a vacuum of 0.3 Torr with an Ar flow to facilitate $\mathrm{Cu}$ and $\mathrm{Au}$ interdiffusion. It was then further annealed at $190^{\circ} \mathrm{C}$ for $10 \mathrm{~h}$ in air for $\mathrm{Cu}$ oxidation. The sample was immersed in a $0.1 \mathrm{M} \mathrm{HCl}$ solution for $9 \mathrm{~h}$ to remove the oxidized copper. After cleaning with deionized water and drying by nitrogen, a porous $\mathrm{Au}$ film was acquired. As shown in Fig. 1a, a lot of randomly distributed pores with sizes of $20-350 \mathrm{~nm}$ are observed by scanning electron microscopy. A clear view of the nanoporous structures is shown in the enlarged embedded picture.

The absorbance spectrum of this sample in the visible regime was investigated to realize the far-field optical properties. Figure $1 b$ shows the absorbance characteristics of the $20 \mathrm{~nm}$ thick Au film (dashed curve), porous Au film (blue curve) and their difference (gray curve). The differences on spectral measurement of Au film with or without the porous structure reveals absorbance band for long wavelength that cutting at $488 \mathrm{~nm}$ with a broad peak at $635 \mathrm{~nm}$. In the following near-field optical studies, the illumination has been done with red light to match the spectral result for achieving the maximal observable response.

Figure 2 is the schematic of experimental setup for the near-field optical observation. Non-optical shear-force feedback system was used for tip-sample distance stabilization. An NSOM (Aurora3, Veeco) equipped with a tapered Alcoated fiber tip with $80 \mathrm{~nm}$ aperture was operated in illumination mode for the optical measurements of the porous $\mathrm{Au}$ film. The light beam from a wavelength-changeable ion laser (Innova 70c, Coherent) was coupled into the single-mode fiber and illuminating at the vicinity of the tip apex. The polarization of the incident light was subsequently controlled by a quarter-wave plate (10RP54-1, Newport) and a polarizer (extinction ratio of $10^{6}: 1$ ). The NSOM was operated in the CGM, and the tip emitted EM field after interacting with the porous $\mathrm{Au}$ film were collected by an objective lens (40×, $\mathrm{NA}=0.65$, Olympus) for TM measurements or a long working distance objective lens $(20 \times, \mathrm{NA}=0.35$, Olympus $)$ for $\mathrm{RM}$ measurements. The topography and near-field scanning optical images were simultaneously recorded by shear force and photomultiplier tube detection, respectively, for subsequent 
Fig. 1 a SEM image of the porous $\mathrm{Au}$ film. b Light absorbance from the porous $\mathrm{Au}$ film compared to pure Au film

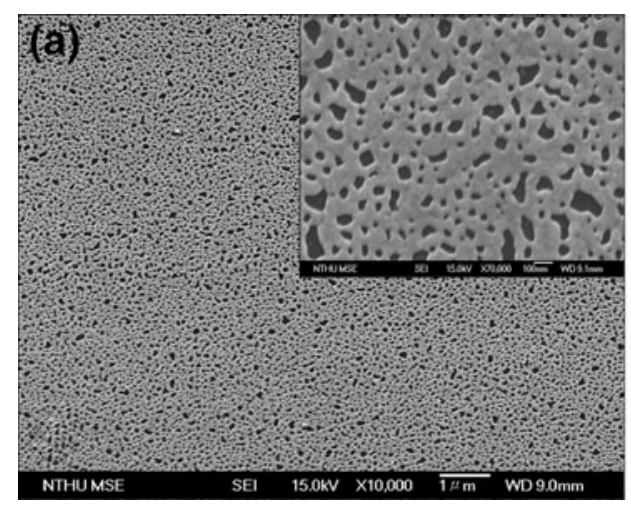

analysis. In addition, the use of illumination mode allows for studying near-field properties of individual points without the interference from the whole sample lighting (collection mode).

\section{Results and Discussion}

Figure 3 a shows a $2 \times 2 \mu \mathrm{m}$ topography of the porous $\mathrm{Au}$ film and Fig. $3 b$ shows a simultaneously recorded RM-NSOM image at $\lambda=632.8 \mathrm{~nm}$. It is clear that the spatial resolution of both images can get down to about $30 \mathrm{~nm}$. The topographic image can provide such a high resolution due to the presence of small protrusive metal grains around the aperture of the probe apex. But the latter is clearly inconsistent with the theory of near-field optics that predicts only about $80 \mathrm{~nm}$ resolution while using an $80 \mathrm{~nm}$ aperture probe. This extraordinary phenomenon obviously allows us to confirm that the near-field optical image of the porous Au film was coupled with undesired artifacts.

In general, artifacts are extremely complicated and require numerical simulation for correction. However, in our special case, they can be removed with a simple approach. Taking into account the influences of our sample, we can

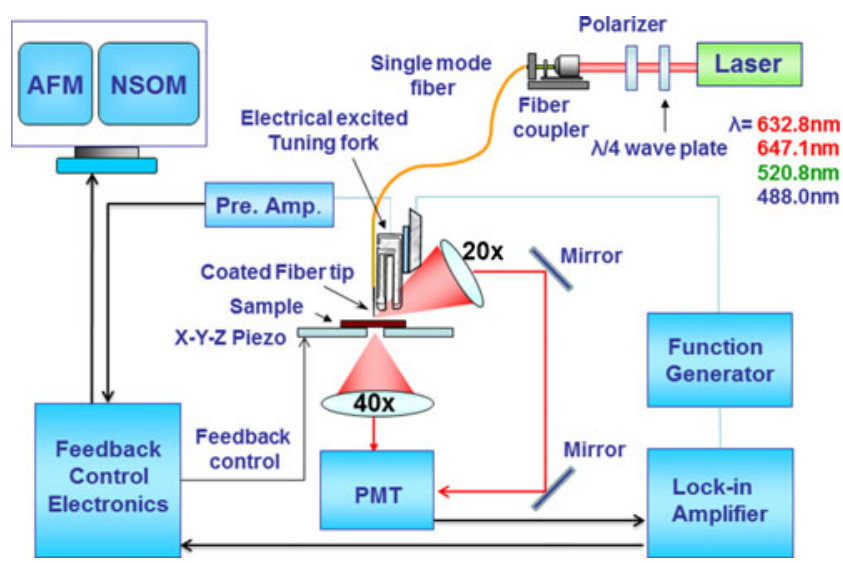

Fig. 2 Experimental setup of the near-field scanning optical microscopy first neglect the artifacts coming from structural scattering [14] because the porous Au film with a roughness of $4 \mathrm{~nm}$, which was measured with the AFM (Veeco, D3100), can be treated as a weakly scattering structure. Second, the quartz substrate with its low reflectivity can also decrease the contributions of artifacts originating in reflection measurement [14]. However, considering the influences of the NSOM operating mode [13-17], the optical signals measured in an off-axis mode will possibly be dominated by the $z$-motion artifacts. This non-optical information caused by constant-gap control can give rise to topographic crosstalk and couple to the purely optical image. We thus can see a lot of localized topographic features with abnormal resolution in Fig. 3b. In previous studies [13-16], first-order perturbation theory indicates that the content of captured near-field optical image is a superposition of the background signals, topographic artifacts, and optical information. These findings allow us to simplify the problem and filter most of the topographic artifacts from the NSOM image of a homogenous sample by making a subtraction, and the correct optical information can then be approximately extracted.

Figure $3 \mathrm{c}$ shows the result acquired by subtracting the topographic image (Fig. 3a) from NSOM image (Fig. 3b). For this process, it was necessary to change the unit of $z$-axis in the topography image (Fig. 3a) from nanometer to photovoltage. It is reasonable that each artifactual feature in NSOM image can coincide perfectly with the topographic feature in topographic image without any plane shifts, even when in practice the accurate optical sensor and effective force sensor on $100 \mathrm{~nm}$ Al-coated fiber probe are always at different positions. With that, the subtraction seemed justified as the amplitudes of artifactual signals induced by $z$-motion in NOSM image are always proportional to the amplitudes of electrical signals captured by the shear-force feedback detection. Thus, by careful itinerary adjustment of the proportionality between the features in Fig. 3a and topographic artifacts in Fig. $3 \mathrm{~b}$ and then by subtraction, the correct near-field optical information was eventually obtained and the undesired artifacts could be eliminated approximately. 
Fig. 3 a Topographic and b RM-NSOM image of the porous $\mathrm{Au}$ film, $\mathbf{c}$ artifact-filtered image of image (b), $\mathbf{d}$ artifactfree TM-NSOM image
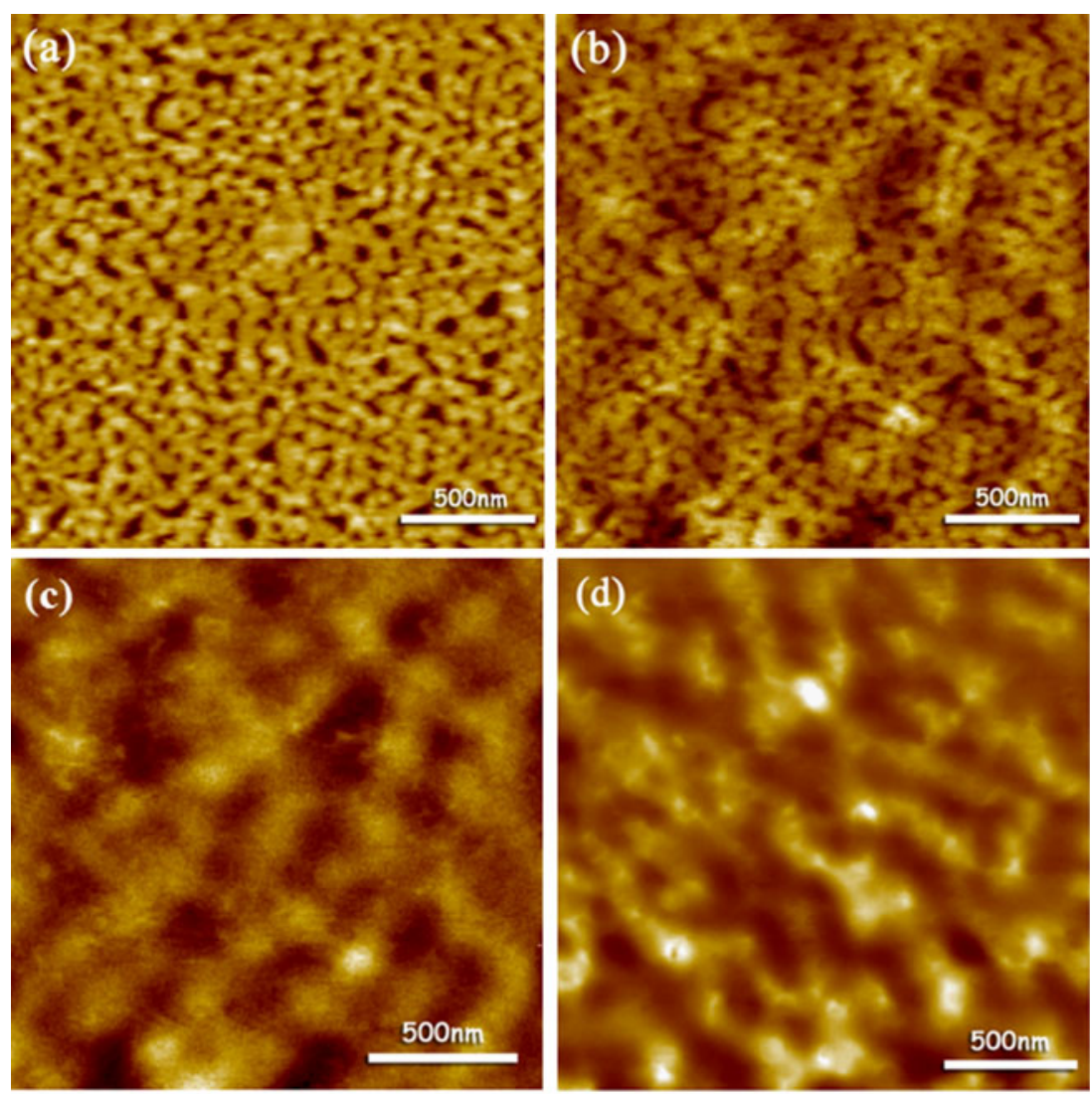

Figure $3 \mathrm{~d}$ shows a TM optical image measured from another area of the porous Au film. This on-axis measurement can be regarded as an artifact-free operating mode. By comparing this image with Fig. 3c, we can easily observe that the two alike distributions of the EM field of these images are displayed with a comparable resolution of about $80-100 \mathrm{~nm}$. The slight difference in contrast in favor of Fig. 3d originates from the difference in light collection efficiency of the two operating modes.

As has been shown in Fig. 3c, the EM field distribution in the near-field optical image of the porous Au film is not uniform. The intensity contrast can neither be explained by artifacts or corresponding features in the topographic image. The contrast provides evidence for plasmonic influences and can be explained as follows. When the fiber probe is being scanned on the Au film surface, the randomly distributed pores and rough structures on the porous Au film help to fulfill the dispersion relation and thereby generate SPPs. Two situations should be considered and are illustrated in Fig. 4. In Fig. 4a, the light from the fiber probe is incident on a nanopore and the optical energy is absorbed by the metal surrounding the nanopore. Spatially extended SPPs are generated and the collective far-field optical properties are similar to LSPs $[25,26]$. This LSP like characteristic from a nanohole is more accurately termed as hole plasmon (HP) [25], and the EM excitation in this case is thus referred to the generation of HPs. This process leads to a decrease of transmitted and reflected light intensities from the region underneath the fiber probe and accounts for the appearance of the dark shady areas in Fig. 3c.

The second case is illustrated in Fig. 4b. When the light from the probe is incident directly on a rough metal structure, SPPs are again generated but spatially confined. They are easily scattered to the far-field via direct scattering or SPP decoupling [32]. The EM excitation in this case is referred to the generation of SPPs. The transmitted and the reflected optical intensities are therefore stronger, which explains the observed bright areas in Fig. 3c. It should be stressed that the two situations are simplified explanation of the plasmonic contrast. The sizes of the pores or the rough structures affect the generation of SPPs significantly and consequently also the transmitted or reflected light intensities. For example, the SPP generation becomes inefficient if the nanopore is large in the first situation. The transmitted light intensity actually becomes high and appears bright in the optical image. Additionally, there are random interferences and multiple couplings of plasmons present. These problematic interactions make a point-by-point inspection of the optical material and structure related properties of the sample impossible.

Owing to the wavelength-dependence characteristic of the porous Au film (see Fig. 1b), the incident wavelength 
Fig. 4 Illuminated region of the sample in near-field with a HPs effect and $\mathbf{b}$ light scattering or SPP decoupling effect

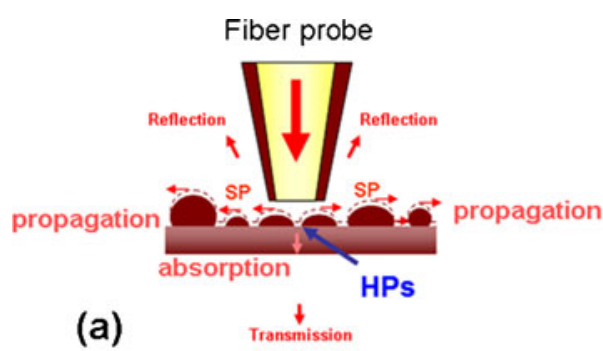

was varied to investigate the plasmonic influences on the near-field distribution. Figure 5 a shows a $5 \times 5 \mu \mathrm{m}$ topographic image of the porous $\mathrm{Au}$ film and Fig. 5b-d reveal simultaneously recorded TM optical images at a wavelength of $488,647.1$, and $520.8 \mathrm{~nm}$, respectively. Note that the circles designate identical locations. As can be seen, Fig. 5b and $\mathrm{d}$ are similar and the bright spots in the two images are directly correlated with pores in the topographic image Fig. 5a. Therefore, the plasmonic influences on the nearfield distribution are negligible at these two wavelengths. On the other hand, Fig. 5c reveals a very different field distribution and resembles Fig. 3c. From Fig. 1b, the absorbance difference between the porous film and the plain film gradually increases after $500 \mathrm{~nm}$ and becomes appreciable after $600 \mathrm{~nm}$. This trend is consistent with the results in Fig. 5b-d. It can thus be inferred that the plasmonic influences on the near-field field distribution appears only when the exciting light is tuned to a proper wavelength. It is also clear the plasmonic effects in a near-field image strongly obscure the optical information from the morphological or material dielectric contrast of the sample. Alternatively, plasmonic effects should be avoided in a near-field optical investigation of morphological or material dielectric contrast, which can be done at a suitable wavelength.

As has been mentioned previously, the intensity contrast in a near-field optical image has contributions from morphology, material dielectric constant, and plasmonic influences. Since the plasmonic influences are polarizationdependent, it is possible to identify plasmonic or nonplasmonic regions on the porous Au film by varying the incident polarization. For this purpose, the laser source was tuned to the wavelength of $647.1 \mathrm{~nm}$ (same wavelength in Fig. 5c) and made linearly polarized. The light emitted from the fiber probe was also linearly polarized with a typical extinction
Fig. 5 A $5 \times 5 \mu \mathrm{m}$ scanning images of the porous Au film: a topographic image, $\mathbf{b}-\mathbf{d}$ corresponding TM-NSOM images at $\lambda=488,647.1$, $520.8 \mathrm{~nm}$, respectively. The circles designate identical locations
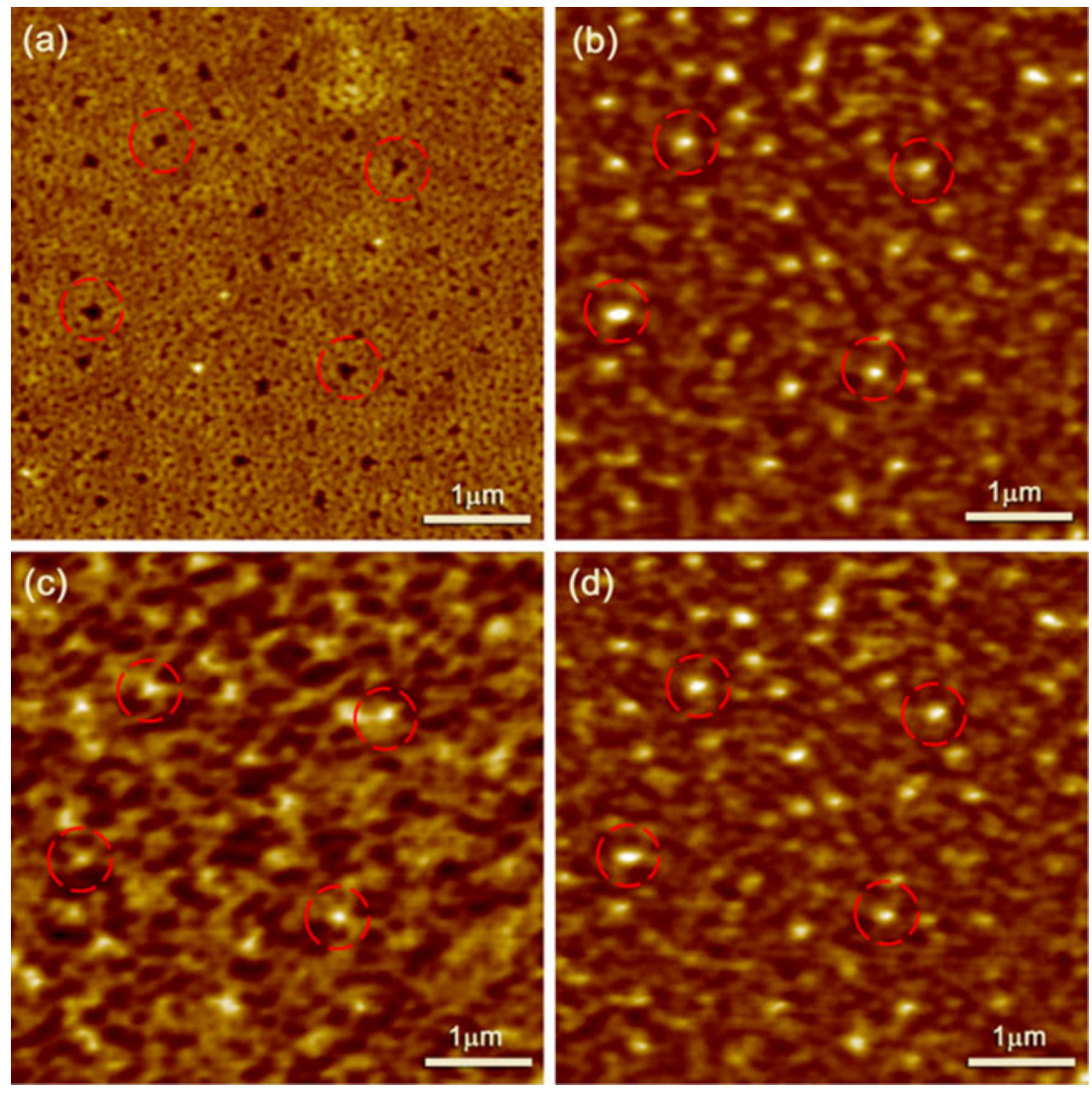
ratio of around $20[12,33,34]$. Figure 6 a shows a $750 \times 750 \mathrm{~nm}$ topographic image of the porous Au film and Fig. $6 b-d$ are simultaneously recorded $\mathrm{TM}$ optical images at $0^{\circ}, 90^{\circ}$, and $45^{\circ}$ polarizations, respectively.

Based on the observed polarization-dependent contrast in the optical images, different plasmonic regions can be classified. To simply the discussion, the optical intensity in a region will be described as high, average, or low. In the region indicated by the white circle, the optical intensity is low and remains unchanged when the polarization is varied. Since this region is accompanied with tiny pores from Fig. 6a, the intensity should be high if the morphological contrast is the main contribution. The observed low intensity in this region consequently exemplifies that it is an example of the generation of HPs as is illustrated in Fig. 4a. The generation process in this region, however, is insensitive to the incident polarization and likely to be due to the round shape of the nanopores.

On the other hand, the intensity in the region indicated by the red hexagon is high at the polarization of $0^{\circ}$ or $45^{\circ}$ and average at $90^{\circ}$. Therefore, this region is an example of the generation of SPPs caused by protruding nanostructures as is illustrated in Fig. 4b. The generation process is now polarization-dependent, which is weak at $90^{\circ}$, and can be attributed to the shape or orientation of the protruding nanostructures. Another similar case is the region indicated by the blue square, but the intensity is now high at all three polarizations although slightly lower at $90^{\circ}$. Finally, the region indicated by the dashed green circle corresponds to a large nanopore in Fig. 6a and the optical intensity is basically high although lower than those in the regions indicated by the red hexagon and the blue square. The optical contrast in this region simply originates from morphological contrast and bears little plasmonic influences.

\section{Conclusions}

In conclusion, we have observed topographic artifacts and surface plasmon influences on the near-field optical images of a porous Au film experimentally. Under tip illumination, topographic artifacts are found to be present in an RM optical image but not in a TM image. It is demonstrated (in our special case) that by subtracting the topographic image from the optical image, the $z$-motion artifact problem in an RM-NSOM image can be filtered and the correct optical information can be extracted approximately. On the other hand, surface plasmon influences are present in both reflection and transmission modes. The near-field optical interference due to surface plasmon excitation and interaction is verified by using three excitation wavelengths of 488 , 647.1 , and $520.8 \mathrm{~nm}$. The result shows that the plasmonic
Fig. 6 A $750 \times 750 \mathrm{~nm}$ scanning images of the porous Au film: a topographic image, b-d corresponding TM-NSOM images measured with polarizations of $0^{\circ}, 90^{\circ}$, and $45^{\circ}$, respectively. The red hexagon and blue square indicate the representative regions of the generation of SPPs. The white circle indicates a region of the generation of HPs and the dashed green circle indicates a region without plasmonic influence
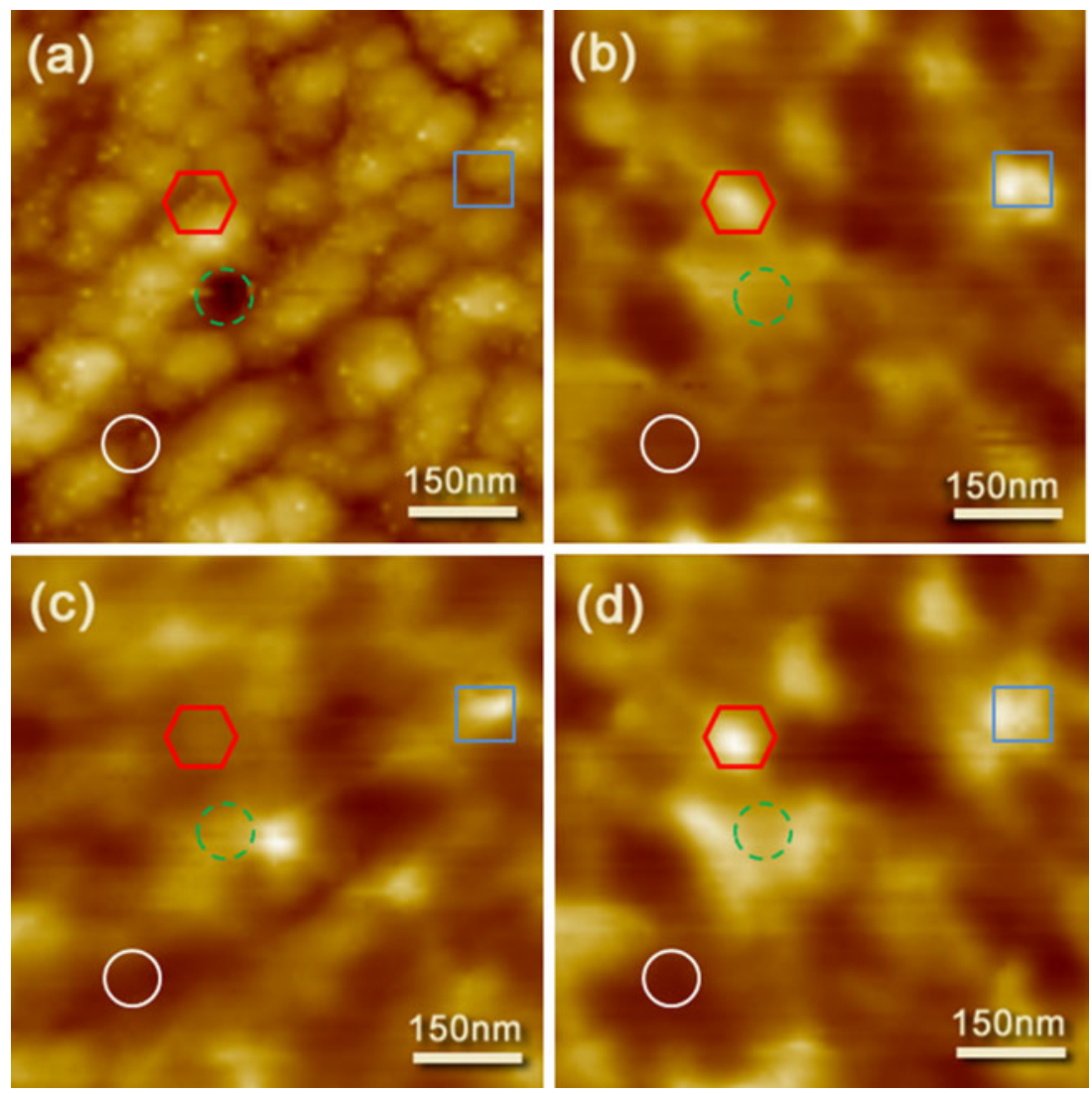
effects exist only in the optical image obtained at the wavelength of $647.1 \mathrm{~nm}$, which correlates well with the far-field optical spectrum measurement, and these effects strongly obscure the optical information from the morphological contrast. High-resolution TM-NSOM measurements at $647.1 \mathrm{~nm}$ with varied polarizations of $0^{\circ}, 90^{\circ}$, and $45^{\circ}$ have been conducted to identify plasmonic and nonplasmonic regions on the sample. Plasmonic regions due to the generation of HPs or SPPs have been found. For the first case, HPs are generated in regions with tiny pores and the optical intensities are low and seemingly polarization independent. For the second case, SPPs are generated in regions with protruding nanostructures and the optical intensities are high and polarization dependent. On the other hand, nonplasmonic regions correspond to large pores and the optical intensities are basically high. The exploration of plasmonic influences in near-field optical images presented in the present work should be able to provide valuable insights in the interpretation of near-field optical results.

\section{References}

1. Pohl DW, Denk W, Lanz M (1984) Optical stethoscopy: image recording with resolution $\lambda / 20$. Appl Phys Lett 44:651-653

2. Betzig E, Finn PL, Weiner JS (1992) Combined shear force and nearfield scanning optical microscopy. Appl Phys Lett 60:2484-2486

3. Reddick RC, Warmack RJ, Ferrell TL (1989) New form of scanning optical microscopy. Phys Rev B 39:767-770

4. Tsai DP, Lu YY (1998) Tapping-mode tuning fork force sensing for near-field scanning optical microscopy. Appl Phys Lett 73:2724-2726

5. Betzig E, Trautman J (1992) Near-field optics: microscopy, spectroscopy, and surface modification beyond the diffraction limit. Science 257:189-195

6. Tsai DP, Lin WC (2000) Probing the near fields of the superresolution near-field optical structure. Appl Phys Lett 77:1413-1415

7. Tsai DP, Yang CW, Lin WC, Ho FH, Huang HJ, Chen MY, Tseng TF, Lee CH, Yeh CJ (2000) Dynamic aperture of near-field super resolution structures. Jpn J Appl Phys 39:982-983

8. Moskovits M (1985) Surface-enhanced spectroscopy. Rev Mod Phys 57:783-826

9. Emory R, Nie S (1997) Near-field surface-enhanced Raman spectroscopy on single silver nanoparticles. Anal Chem 69:2631-2635

10. Weeber JC, Krenn JR, Dereux A, Lamprecht B, Lacroute Y, Goudonnet JP (2001) Near-field observation of surface plasmon polariton propagation on thin metal stripes. Phys Rev B 64:045411

11. Imura K, Nagahara T, Okamoto H (2005) Near-field optical imaging of plasmon modes in gold nanorods. J Chem Phys 122:154701

12. Hecht B, Sick B, Wild UP, Decker V, Zenobi R, Martin OJF, Pohl DW (2000) Scanning near-field optical microscopy with aperture probes: fundamentals and applications. J Chem Phys 112:77617774

13. Hecht B, Bielefeldt H, Inouye Y, Pohl DW, Novotny L (1997) Facts and artifacts in near-field optical microscopy. J Appl Phys 81:2492-2498
14. Carminati R, Madrazo A, Nieto-Vesperinas M, Greffet J-J (1997) Optical content and resolution of near-field optical images: influence of the operating mode. J Appl Phys 82:501-509

15. Gucciardi PG, Colocci M (2001) Different contrast mechanisms induced by topography artifacts in nearfield optical microscopy. Appl Phys Lett 79:1543-1545

16. Valle PJ, Greffet J-J, Carminati R (1999) Optical contrast, topographic contrast and artifacts in illumination-mode scanning nearfield optical microscopy. J Appl Phys 86:648-656

17. Wang X, Fan Z, Tang T (2005) Simulation of topographic images and artifacts in illumination-mode scanning-near-field optical microscopy. J Opt Soc Am A 22:2730-2736

18. Jordan CE, Stranick SJ, Richter LJ, Cavanagh RR (1999) Removing optical artifacts in near-field scanning optical microscopy by using a three-dimensional scanning mode. J Appl Phys 86:27852789

19. Raether H (1988) Surface plasmons on smooth and rough surfaces and on gratings. Springer, Berlin

20. Kawata S (2001) Near-field optics and surface plasmon polaritons. Springer, Berlin

21. Markel VA, George TF (2001) Optics of nanostructured materials. Wiley, New York

22. Quinten M, Leitner A, Krenn JR, Aussenegg FR (1998) Electromagnetic energy transport via linear chains of silver nanoparticles. Opt Lett 23:1331-1333

23. Huang HJ, Yu CP, Chang HC, Chiu KP, Chen HM, Liu RS, Tsai DP (2007) Plasmonic optical properties of a single gold nano-rod. Opt Express 15:7132-7139

24. Ebbesen TW, Lezec HJ, Ghaemi HF, Thio T, Wolff PA (1998) Extraordinary optical transmission through sub-wavelength hole arrays. Nature (London) 391:667-669

25. Park TH, Mirin N, Lassiter JB, Nehl CL, Halas NJ, Nordlander P (2008) Optical properties of a nanosized hole in a thin metallic film. ACS Nano 2:25-32

26. Prikulis J, Hanarp P, Olofsson L, Sutherland D, Käll M (2004) Optical spectroscopy of nanometric holes in thin gold films. Nano Lett 4:1003-1007

27. Parsons J, Hendry E, Burrows CP, Auguié B, Sambles JR, Barnes WL (2009) Localized surface-plasmon resonances in periodic nondiffracting metallic nanoparticle and nanohole arrays. Phys Rev B 79:073412

28. Liu WC, Tsai DP (2001) Optical tunneling effect of surface plasmon polaritons and localized surface plasmon resonance. Phys Rev B 65:155423

29. Ho FH, Lin WY, Chang HH, Lin YH, Liu WC, Tsai DP (2001) Nonlinear optical absorption in the AgOx-type super-resolution near-field structure. Jpn J Appl Phys 40:4101-4102

30. Lin WC, Kao TS, Chang HH, Lin YH, Fu YH, Wu CT, Chen KH, Tsai DP (2003) Study of a super-resolution optical structure: polycarbonate/ZnS-SiO2/ZnO/ZnS-SiO2/Ge2Sb2Te5/ZnS-SiO2. Jpn J Appl Phys 42:1029-1030

31. Zhou H, Jin L, Xu W (2007) New approach to fabricate nanoporous gold film. Chin Chem Lett 18:365-368

32. Devaux E, Ebbesen TW, Weeber JC, Dereux A (2003) Launching and decoupling surface plasmons via micro-gratings. Appl Phys Lett 83:4936-4938

33. Eggersa G, Rosenbergera A, Helda N, Münnemanna A, Güntherodta G, Fumagallib P (1998) Scanning near-field magneto-optic microscopy using illuminated fiber tips. Ultramicroscopy 71:249-256

34. Veerman JA, Otter AM, Kuipers L, van Hulst NF (1998) High definition aperture probes for near-field optical microscopy fabricated by focused ion beam milling. Appl Phys Lett 72:3115-3117 